\title{
Investigation of the Interaction between a Helium Plasma J et and Conductive (Metal)/ Non-Conductive (Dielectric) Targets
}

\author{
Aboubakar Koné, ${ }^{*}$ Florent P. Sainct, Cristina Muja, Bruno Caillier, \\ \& Philippe Guillot
}

Laboratoire Diagnostics des Plasmas Hors Equilibre, INU Champollion, Université de Toulouse, France

*Address all correspondence to: Aboubakar Koné, Laboratoire Diagnostics des Plasmas Hors Equilibre, INU Champollion, Université de Toulouse, Place de Verdun 81012 Albi cedex 9, France; Tel.: +33 563486 431; +33 563486 444; or +33563486 445, E-mail: akone@univ-jfc.fr

\begin{abstract}
Recent research in plasma biology proved that atmospheric pressure plasma jets (APPJs) have a biocidal effect, making them a promising alternative to traditional antimicrobial treatments. Working gas and voltage waveform are well-known parameters influencing the biocide efficiency. The type of surface targeted by the plasma jet also affects the biocidal capability of the APPJs, the streamer propagation, and the chemistry involved. The aim of this study is to investigate experimentally the effect of the target conductivity on the plasma characteristics: plasma jet propagation and reactive species production. The results show that the propagation mechanism is modified when the plasma interacts with the target. These modifications depend on the conductivity of the target. The results also demonstrate that the generation of reactive oxygen and nitrogen species (RONS) is influenced by the target's conductivity. This study shows that the assessment of RONS generated by APPJs should be performed as close as possible to the real applications conditions.
\end{abstract}

KEY WORDS: reactive species, atmospheric pressure plasma jet, surface ionization wave, streamer, copper target, glass target

\section{INTRODUCTION}

In the past few decades, atmospheric pressure plasma jets (APPJs) have emerged as a promising tool for numerous fields, ${ }^{1}$ such as plasma medicine or biomedical applications. ${ }^{2,3}$ Many device configurations have been developed to generate the APPJs, ${ }^{1-3}$ all sharing common characteristics: a flux of noble gas ( $\mathrm{He}, \mathrm{Ne}, \mathrm{Ar}$, or mixture) going through a vessel, symmetrical (tube) or not (diameter discontinuity), a high voltage applied between electrodes with various waveform shape (e.g., sinus, rectangular, pulse), and a propagation of the ionization waveform outside the vessel, which leads to a nonequilibrium plasma at atmospheric pressure. After several experimental and numerical studies ${ }^{4-9}$ the physics of the propagation of the APPJs in free air is now well understood. The propagation in free air of the plasma is mainly a streamer guided by the mixing layer of the noble gas and ambient air. Depending on the experimental conditions, the discharge could spread over $10 \mathrm{~cm}$ from the gas nozzle. Most of the studies are performed in free air conditions with helium as the working gas. However, all the research- 
ers working on APPJs have observed that several modifications occurred on the APPJ's propagation when the plasma jet interacts with the surface of the target. As the discharge produced reactive radical species, it is important to understand the effect of surface on this production. Early investigations should focus on understanding the "remote" delivery of radical or charged species on the treated surfaces. With this goal in mind, some recent articles studied the interaction between the plasma jet and various surfaces, such as wafers, dielectric, glass, agar, or tissue in helium as noble gas. ${ }^{10-14}$ In this work, we are going to present our experimental investigations on the spatial localization of species production for an APPJ in helium and this interaction with surface.

This study will help to understand the mechanisms of radical production associated with the surface interactions. Two different surfaces have been chosen as targets, a floating copper plate and a dielectric plate.

\section{MATERIALS AND MEIHODS}

The apparatus (Fig. 1) used in this investigation was used in a previous study. ${ }^{15}$ The system is a polypropylene dielectric barrier discharge device. It is asymmetrical, like a syringe with a cylindrical part (diameter of $38 \mathrm{~mm}$ and a length of $65 \mathrm{~mm}$ ) and a conical part (diameter of $7 \mathrm{~mm}$ to $3 \mathrm{~mm}$ and length of $43 \mathrm{~mm}$ ). The grounded electrode, 22 $\mathrm{mm}$ wide, wraps around the cylinder whereas the high-voltage electrode, $38 \mathrm{~mm}$ wide, wraps around the cone. These two electrodes are made of aluminum foil (3M, tape 425). High-purity helium $(99.9999 \%$, Linde) is used as the working gas. The gas flow rate is

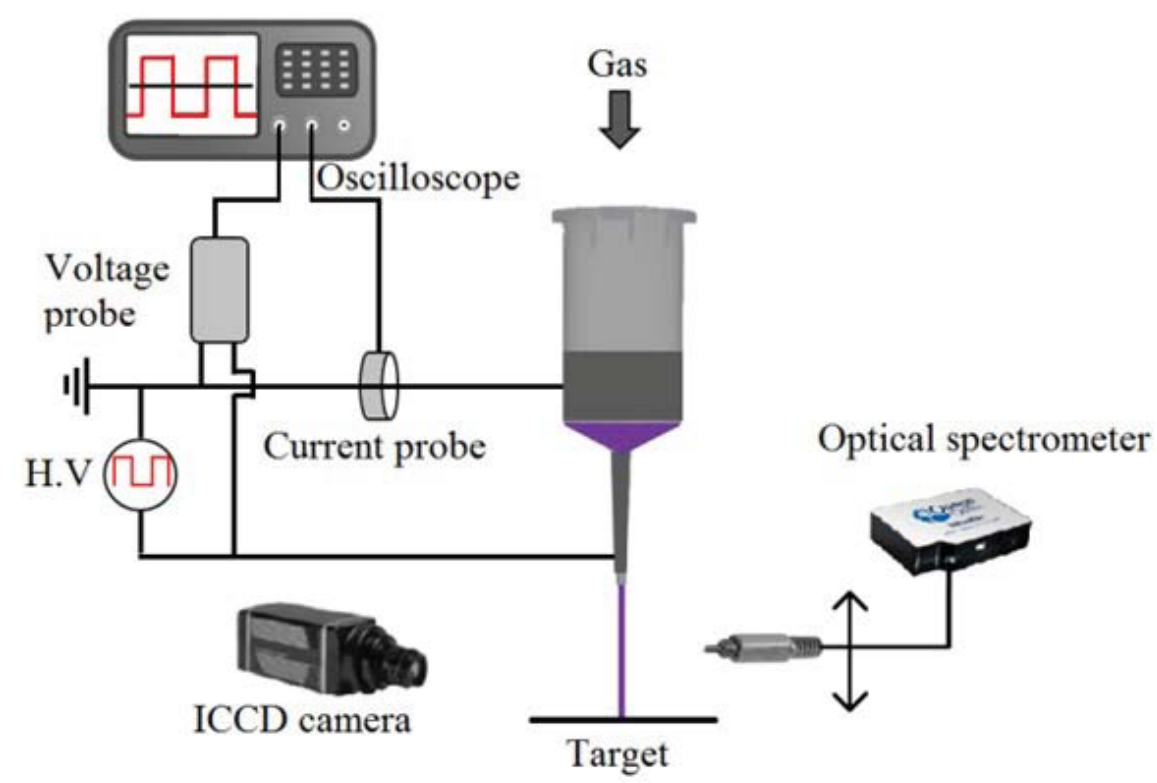

FIG. 1: Schematic description of the experimental setup 
controlled by a volume flow meter (Bronkhosrt, F-201CV). In this study, the gas flow is fixed at $2 \mathrm{SLM}$. A square voltage of $4 \mathrm{kV}_{\mathrm{PP}}$ at $20 \mathrm{kHz}$ with a duty cycle of $50 \%$ is applied to the electrodes to ignite the plasma. A high-voltage probe (Tektronix, P6015A) and a current probe (Pearson, 6585) are used to monitor applied voltage and discharge current on a digital oscilloscope (Tektronix, TDS 7104).

Optical emission spectra were acquired via an optical fiber (Ocean Optics, QP6002-SR), placed perpendicular to the jet flux at $5 \mathrm{~cm}$ away and coupled with an optical spectrometer (Ocean Optics, HR 2000+). The spectrometer's range allows measurements from $200 \mathrm{~nm}$ to $1,100 \mathrm{~nm}$ with a resolution of $0.35 \mathrm{~nm}$. In order to perform a spatial evolution of the optical emission along the plasma plume, the optical fiber was mounted on a translation rail with a displacement step of $1 \mathrm{~mm}$. An intensified coupled charge device (ICCD) camera (Princeton Instruments, PI-MAX) was used with $10 \mathrm{nsec}$ exposure time and gain of 225 to observe the plasma discharge dynamics. A digital camera (Konica) was used to get pictures of the plasma jet in true color.

A copper plate (45 mm long x $30 \mathrm{~mm}$ wide $\mathrm{x} 1 \mathrm{~mm}$ thick) was used as the conductive target. The copper plate was not connected to the ground (floating potential). A glass plate (45 $\mathrm{mm} \times 25 \mathrm{~mm} \times 1 \mathrm{~mm}$ ) was used as the nonconductive target.

\section{RESULTS AND DISC USSION}

The plasma jet is generated without target (free jet), with a copper plate used as a conductive target, and a glass target used as a nonconductive target or dielectric target. The experimental conditions were about $40 \%$ relative humidity and $22^{\circ} \mathrm{C}$. It's important to keep in mind that the copper and glass targets were not connected or placed on support connected to the ground. The present study is not focused on the plasma inside the source, but rather the plasma effluent (plasma jet) in the surrounding air.

\section{A. Photographic Obsenvations}

Figure 2(a) shows an image of a free plasma jet. The purple color is characteristic of visible emissions of a helium plasma jet. The intensity of the jet is higher at the jet device outlet and decreases slowly. The jet length is about $35 \mathrm{~mm}$. Figure 2(b) and (c) show the change in the plasma jet behavior when it interacts with glass and copper targets, and two very different responses are observed. Indeed, when the plasma jet interacts with the glass target (Fig. 2[b]), the plasma seems faintly spread on the glass surface with no significant change in the plasma jet intensity. This behavior completely differs when a copper plate is used as the target (Fig. 2[c]). The plasma jet looks like a "luminous fixed tip," in which the intensity increases most at the region close to the copper surface. Similar observations were recently made in an etching process treatment. ${ }^{16}$

Volume 7, Issue 4, 2017 


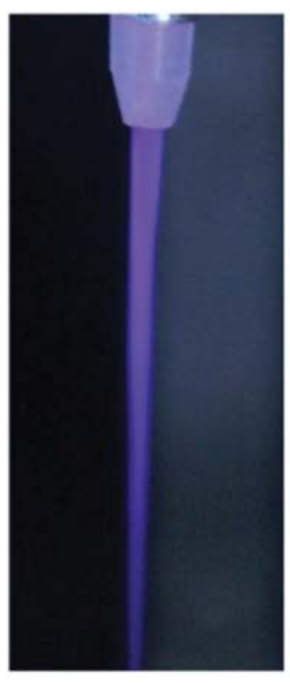

(a)

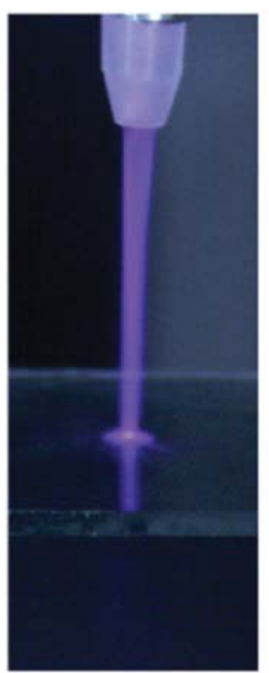

(b)

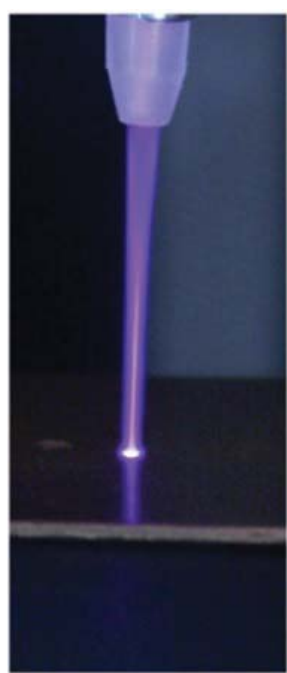

(c)

FIG. 2: Pictures of the plasma jet: (a) free jet mode, (b) with glass target, (c) with copper target. Both copper and glass targets were $15 \mathrm{~mm}$ from the source nozzle

\section{B. ICCD Camera Measurement}

\section{The Free J et Mode}

To better understand the preliminary observations made previously from Fig. 2, the plasma jet dynamic outside the source was observed in free jet. Pictures were taken with an exposure time of $10 \mathrm{nsec}$, every $10 \mathrm{nsec}$. These experiments were performed for two voltage transitions: (1) a voltage rise phase (from $-2 \mathrm{kV}$ to $2 \mathrm{kV}$ with a rise time of 100 nsec [positive transition]), and (2) a voltage fall phase (from $2 \mathrm{kV}$ to $-2 \mathrm{kV}$ with a fall time of $100 \mathrm{nsec}$ [negative transition]). In each case, the beginning of the time count for ICCD imaging was synchronized to correspond with the beginning of the voltage transition phase. For convenience, we defined the plasma jet generated in positive transition and negative transition, respectively, as a positively excited plasma jet (PEPJ) and a negatively excited plasma jet (NEPJ).

The dynamics of PEPJs and NEPJs are illustrated in Figs. 3(a) and 3(b), respectively. Two different propagation dynamics can be observed. The PEPJ consists of an ionization wave that resembles a "luminous plasma head" (Fig. 3[a]). This structure moves away from the plasma source nozzle, leaving behind a less luminous channel. During its propagation, a change in shape and velocity of the "plasma head" volume is observed. For example, since the exit the plasma head moves the first $15 \mathrm{~mm}$ in 270 nsec, which corresponds to a mean velocity of $55 \mathrm{~km}_{-} \mathrm{sec}^{-1}$. Next, both velocity and 
emission intensity decrease until the plasma head is no longer visible with the ICCD camera. In this second part, the plasma head moves about $24 \mathrm{~mm}$ in $600 \mathrm{nsec}$, which corresponds to a mean velocity of about $40 \mathrm{~km}-\mathrm{sec}^{-1}$.

The NEPJ is composed of a continuous glow ionization channel. Compared to the PEPJ, the NEPJ emissions are less intense. This leads to an increased number of ICCD camera accumulations ( $10^{4}$ accumulations $)$ compared to those for PEPJs $\left(1.5 \times 10^{3}\right.$ accumulations). The structure is more complex because of the continuous form of the ionization wave. However, a change in the ionization wave shape from the plasma source nozzle is clearly observed during the first $800 \mathrm{nsec}$. In fact, these first $800 \mathrm{nsec}$ correspond to the propagation of the ionization wave. This plasma moves about $10 \mathrm{~mm}$ in 700 nsec, which corresponds to a mean velocity of about $14 \mathrm{~km}-\mathrm{sec}^{-1}$. Then, the propagation of the NEPJ ceases with weak intensity. The results obtained on the propagation and the velocity magnitude for the plasma jets on the two polarity transitions corresponds to what has been described elsewhere in the literature. ${ }^{17-19}$

The first explanation on the dynamics of plasma jets was proposed by Laroussi and Lu in 2006. ${ }^{17}$ They observed some similarities between PEPJs and a model describing the propagation of positive corona streamers proposed by Dawson and Wien. ${ }^{20}$ Laroussi
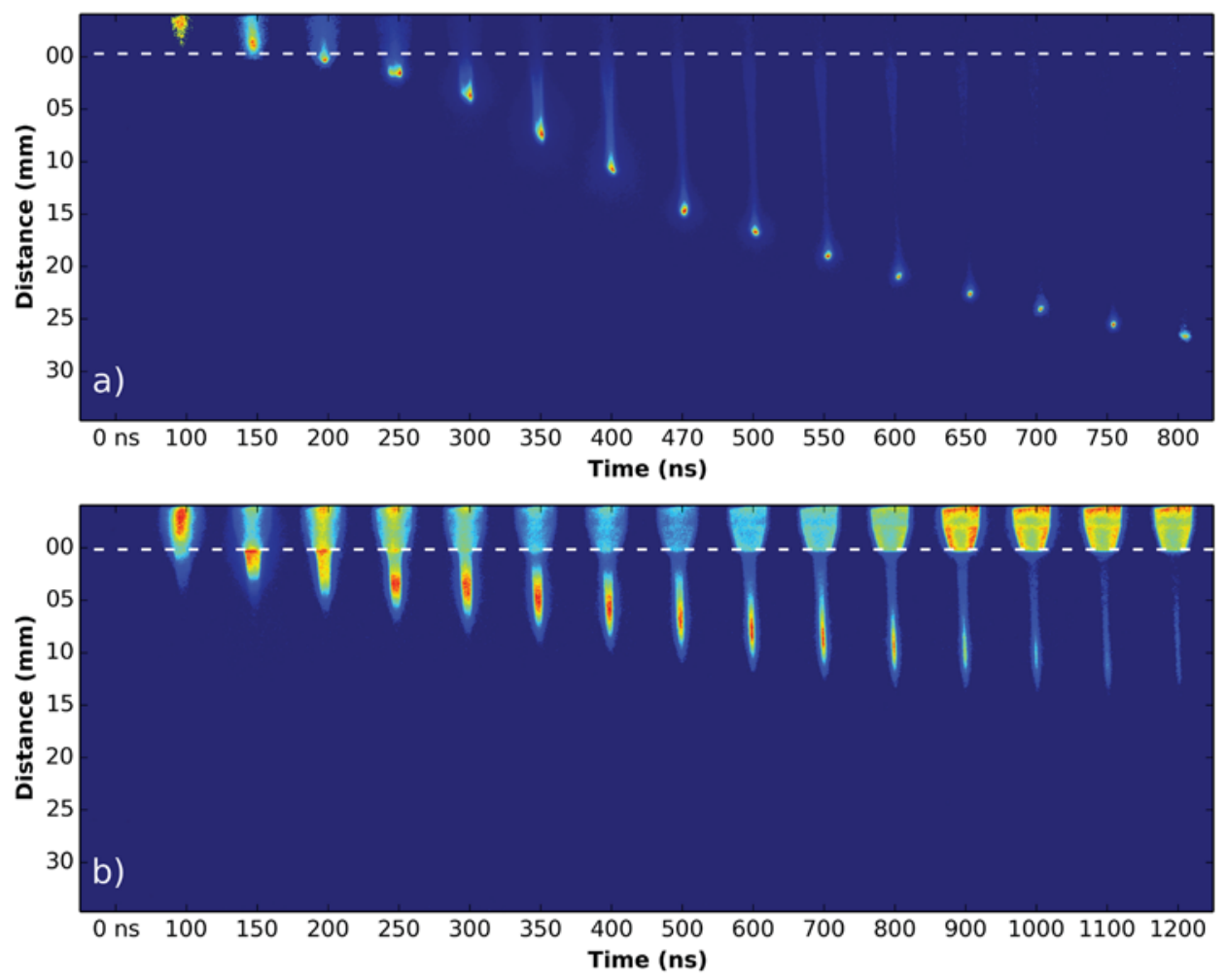

FIG. 3: ICCD measurement: (a) PEPJ $\left(1.5 \times 10^{3}\right.$ accumulations), (b) NEPJ ( $10^{4}$ accumulations). The white dash line indicates the end of the plasma source

Volume 7, Issue 4, 2017 
and $\mathrm{Lu}$ suggested a theory based on photoionization to explain the magnitude of the bullet-like volume propagation velocity. Later, numerous authors, including Naidis performed experiments with streamer standard models, which described the dynamics and the structure of plasma jets for both positive and negative polarities of applied voltage. ${ }^{21}$ They all confirm the similarities between positive streamers and PEPJs on one hand, and negative streamers and NEPJs on the other hand. However, some authors agree with the fact that photoionization plays an essential role in the propagation velocity of streamers, but suggest that photoionization is not necessary to sustain streamers. They propose a background pre-ionization theory as a valuable mechanism to sustain streamers in an air atmosphere. ${ }^{22}$

Although the polarity of the discharge clearly has an important influence on plasma jet dynamics, the mechanisms of propagation of PEPJs and NEPJs are the same. Otherwise, if one takes into account just the velocity and the propagation length, it seems that PEPJs are more suitable than NEPJs for future applications.

\section{Interaction of the Plasma Jet with Targets}

In order to study the modification introduced by a target on plasma jets properties, the previous experiments were carried out, but both copper and glass target were placed 15 $\mathrm{mm}$ from the source exit. Figure 4 shows the ICCD measurement when the copper target interacts with the plasma jet. The behavior is quite different depending on the polarity. For PEPJs (Fig. 4[a]), the plasma head crosses the $15 \mathrm{~mm}$ between the source nozzle and the copper's surface in $260 \mathrm{nsec}$. This shows a mean velocity of about $60 \mathrm{~km}-\mathrm{sec}^{-1} . \mathrm{Im}-$ mediately after the plasma head strikes the copper target, a conductive channel starts to form from the target to the source exit. The full establishment of this channel takes about $50 \mathrm{nsec}$. It is interesting to observe that the increased intensity of the channel matches with the decreased intensity of the plasma head at the copper target surface. Afterward, the channel intensity still decreases from the target to the nozzle. For the NEPJ (Fig. $4[\mathrm{~b}]$ ), the ionization wave strikes the target surface at $720 \mathrm{nsec}$, (mean velocity $24 \mathrm{~km}$ $\mathrm{sec}^{-1}$ ). The maximum intensity part of the ionization wave increases in the direction of the source nozzle from $4 \mathrm{~mm}$ at $720 \mathrm{nsec}$ to $7 \mathrm{~mm}$ at $950 \mathrm{nsec}$. Then, the intensity of the streamer starts to gradually diminish. During this decrease, the maximum intensity part of the ionization wave is still located on the target surface.

The same study made with a glass target is presented in Fig. 5. In this case, the ionization wave in the PEPJ (Fig. 5[a]) exits from the source nozzle at $200 \mathrm{nsec}$ and reaches the surface at $460 \mathrm{nsec}$, which corresponds to a mean velocity of about $60 \mathrm{~km}-\mathrm{sec}^{-1}$. Then, the plasma head volume starts spreading on the glass surface and forms a region of $1 \mathrm{~mm}$ in diameter. The most intense point of this region is located in the center of the plasma head. No significant change in the channel formed behind the plasma head is observed. For the NEPJ (Fig. 5[b]), the results are the same as in free jet mode, because the negative streamer doesn't touch the glass target.

Using a numerical model, Norberg, Johnsen, and Kushner have investigated the interaction between a helium plasma jet and materials with different permittivity, includ- 

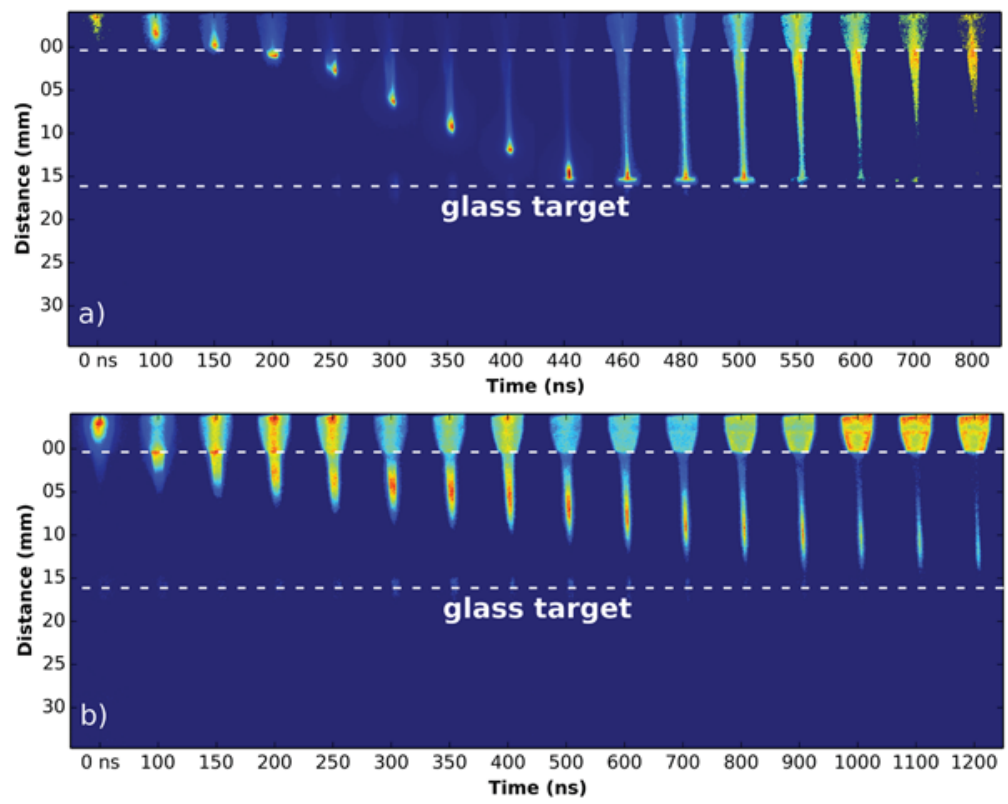

FIG. 4: ICCD measurement: (a) PEPJ ( $10^{3}$ accumulations), (b) NEPJ ( $10^{4}$ accumulations). The copper target is $15 \mathrm{~mm}$ from the syringe nozzle. The white dash lines indicate the syringe nozzle and the location of the copper plate.
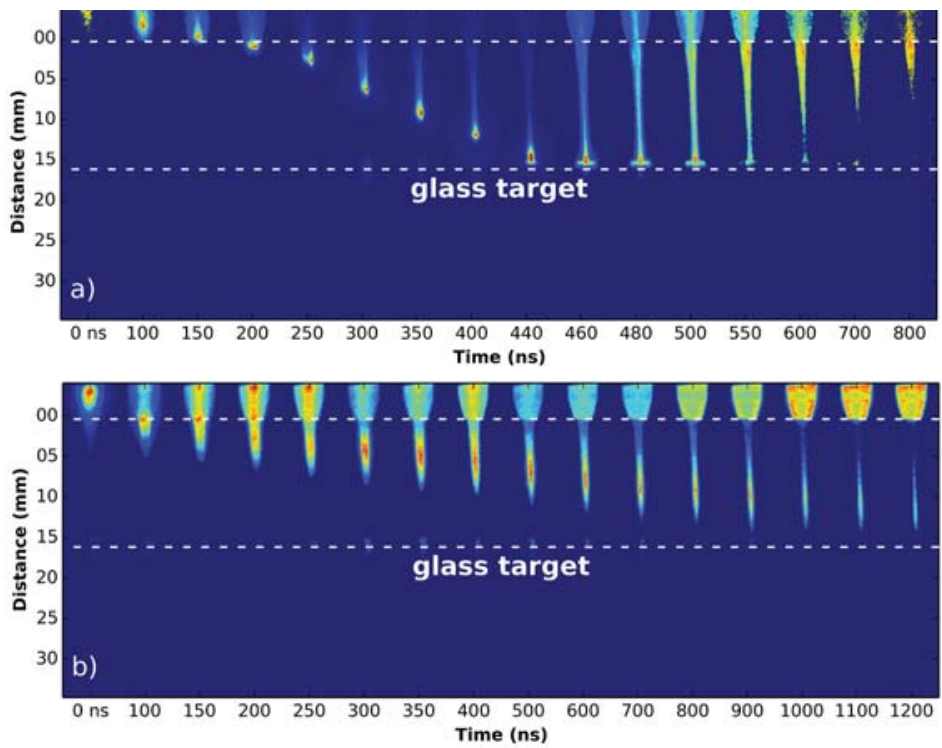

FIG. 5: ICCD measurement: (a) PEPJ ( $10^{3}$ accumulations), (b) NEPJ ( $10^{4}$ accumulations). The glass target is $15 \mathrm{~mm}$ from the syringe nozzle. The white dash lines indicate the syringe nozzle and the location of the glass plate.

Volume 7, Issue 4, 2017 
ing glass and metal. Our results are in good agreement with their study. ${ }^{23}$ In fact, they observed the spreading effect of the ionization wave after the impact with lower permittivity materials such as glass. For PEPJs, they also described the presence of a conduction channel generated after the ionization wave strikes the metal surface. The model shows that when the ionization wave is in contact with the low permittivity materials, it leads to the charging of the material's capacitance. The capacitance charging generates a lateral electric field, which sustains the ionization on the material surface and forms a surface ionization wave (SIW). For a metal surface, the presence of a conductive channel and also the absence of SIW were confirmed. In this case, no capacitance charging occurs when the plasma head reaches the target. This leads to the absence of a horizontal component of the electric field and the formation of a SIW. Yan and Economou also confirmed these results in their numerical study and gave a similar explanation. ${ }^{24}$ Finally, the study by Guaitella and Sobota concerning interactions between plasma jets with dielectric surfaces provides similar results. ${ }^{25}$

The ICCD measurements provide knowledge on the dynamic and the interaction mechanism between plasma jet and the different surfaces. However, this diagnostics tool doesn't reveal the presence and the modification of reactive nitrogen species (RNS) and reactive oxygen species (ROS) generated by the plasma jet. This additional information has been supplied by optical spectroscopic measurements.

\section{Optics Spectrosc opic Measurement}

\section{Optical Spectra}

Optical emission spectroscopy is a noninvasive technique suitable to identify reactive species generated in the plasma jet. First, the optical fiber was placed perpendicularly to the plasma jet's propagating direction, $14 \mathrm{~mm}$ from the source nozzle in free jet mode. The spectrum recorded was done with a 2 -sec integration time and averaging of three measurements. As shown in Fig. 6(a), the most intense emissions of the spectra in free jet mode are located in the UV and near visible regions $(290-500 \mathrm{~nm})$. Theses emissions were identified as transitions provided from the $\mathrm{N}_{2}{ }^{*}$ second positive system $\left(\mathrm{N}_{2}{ }^{*} \mathrm{SPS}\right)$ and $\mathrm{N}_{2}{ }^{+}$first positive system (FPS) of the nitrogen molecule.

$\mathrm{N}_{2}{ }^{2}$ SPS emissions are due to the following processes ${ }^{26}$ :

electron-impact excitation of the $C^{3} \Pi_{u}$ state from the $X^{I} \sum_{g}{ }^{+}$ground state:

$$
\mathrm{N}_{2}\left(X^{l} \sum_{g}^{+}\right)+\mathrm{e}^{-} \rightarrow \mathrm{N}_{2}\left(C^{3} \Pi_{u}\right)+\mathrm{e}^{-}
$$

followed by radiative decay:

$$
\mathrm{N}_{2}\left(C^{3} \Pi_{u}\right) \rightarrow \mathrm{N}_{2}\left(B^{3} \Pi_{g}\right)+h v
$$

mutual collisional quenching of the metastable $\mathrm{A}^{3} \sum_{\mathrm{u}}{ }^{+}$states:

$$
\mathrm{N}_{2}\left(A^{3} \sum_{u}^{+}\right)+\mathrm{N}_{2}\left(A^{3} \sum_{u}^{+}\right) \rightarrow \mathrm{N}_{2}\left(C^{3} \Pi_{u}\right)+\mathrm{N}_{2}\left(X^{1} \sum_{g}^{+}\right)
$$



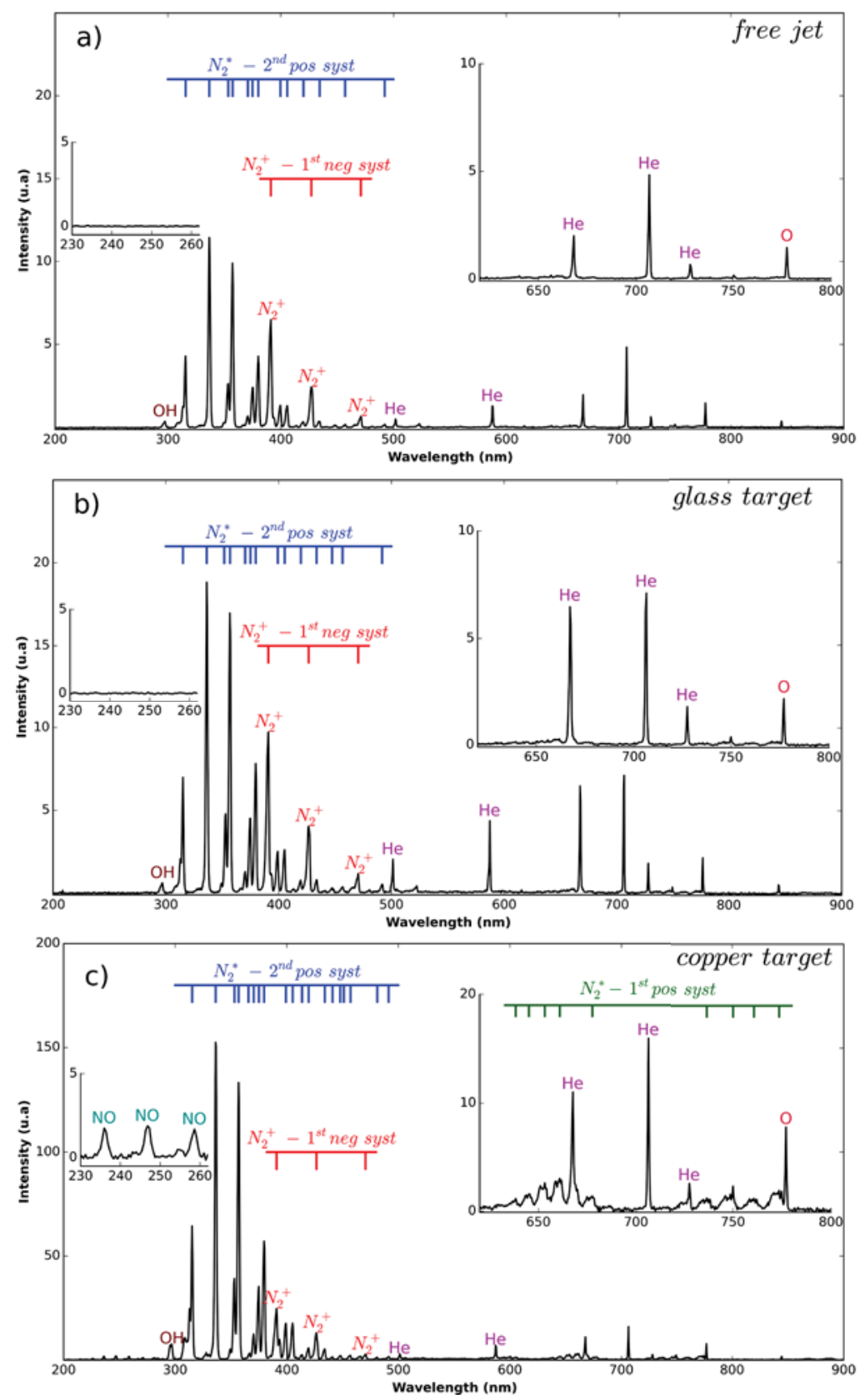

FIG. 6: Optical spectra of plasma jet: (a) free jet, (b) with glass target, (c) with copper target. Both glass and copper targets were $15 \mathrm{~mm}$ from the source nozzle. The spectra were recorded with 2-sec integration time and averaging of three measurements.

Volume 7, Issue 4, 2017 
$\mathrm{N}_{2}{ }^{+}$FPS are attributed to ${ }^{27}$ :

penning ionization with helium metastable:

$$
\mathrm{He}^{*}+\mathrm{N}_{2}\left(X^{1} \sum_{g}^{+}\right) \rightarrow \mathrm{He}+\mathrm{N}_{2}^{+}\left(B^{2} \sum_{u}^{+}\right)+\mathrm{e}^{-}
$$

followed by radiative decay:

$$
\mathrm{N}_{2}^{+}\left(B^{2} \sum_{u}^{+}\right) \rightarrow \mathrm{N}_{2}^{+}\left(X^{2} \sum_{g}^{+}\right)+h v
$$

charge transfer:

$$
\mathrm{He}^{+}+\mathrm{N}_{2}\left(X^{l} \sum_{g}^{+}\right) \rightarrow \mathrm{He}+\mathrm{N}_{2}^{+}\left(B^{2} \sum_{u}^{+}\right)
$$

followed by radiative decay:

$$
\mathrm{N}_{2}^{+}\left(B^{2} \sum_{u}^{+}\right) \rightarrow \mathrm{N}_{2}^{+}\left(X^{2} \sum_{g}^{+}\right)+h v
$$

$\mathrm{OH}$ line emission at $307 \mathrm{~nm}$ observed comes from: water vapor dissociation ${ }^{23}$ :

$$
\mathrm{H}_{2} \mathrm{O}+\mathrm{e} \rightarrow \mathrm{H}+\mathrm{OH}\left(A^{2} \sum^{+}\right)
$$

followed by radiative decay:

$$
\mathrm{OH}\left(A^{2} \sum^{+}\right) \rightarrow \mathrm{OH}\left(X^{2} \Pi\right)+h v
$$

O lines at $777 \mathrm{~nm}$ and $844 \mathrm{~nm}$, from the dissociation of oxygen molecule present in air by: electronic impact ${ }^{28}$ :

$$
\begin{aligned}
& \mathrm{e}^{-}+\mathrm{O}_{2}\left(X^{3} \sum_{g}\right) \rightarrow \mathrm{O}_{2}\left(A^{3} \sum_{u}^{+}\right) \rightarrow \mathrm{O}\left({ }^{3} P\right)+\mathrm{O}\left({ }^{3} P\right)+\mathrm{e}^{-} \\
& \mathrm{e}^{-}+\mathrm{O}_{2}\left(X^{3} \sum_{g}\right) \rightarrow \mathrm{O}_{2}\left(B^{3} \sum_{u}{ }^{+}\right) \rightarrow \mathrm{O}\left({ }^{3} P\right)+\mathrm{O}\left({ }^{1} D\right)+\mathrm{e}^{-}
\end{aligned}
$$

penning dissociation ${ }^{29}$ :

$$
\mathrm{N}_{2}{ }^{*}+\mathrm{O}_{2} \rightarrow \mathrm{N}_{2}+\mathrm{O}+\mathrm{O}
$$

He* lines are the result of the electron impact with helium, with a minimum threshold energy of $19.8 \mathrm{eV} .^{23}$

In a second study, a target was placed at $15 \mathrm{~mm}$ from the source nozzle. The optical fiber was placed at $14 \mathrm{~mm}$ from the source nozzle. This configuration allows the measurement of the target's influence on the plasma jet properties in the region close to the target surface. When glass is used as the target, the spectra obtained (Fig. 6[b]) presents no significant change in the intensity of reactive species compared to the free jet's spectra. Figure 6(c) shows spectra obtained when the copper target was placed in front of the plasma jet. Compared to free jet mode, several differences can be observed. First, an increase in emission intensity for all lines is observed. For example, for the $\mathrm{N}_{2}{ }_{2}$ second positive system, the emission intensities were multiplied by a factor of 10 . In the same time, the presence of the copper target generates species not observed in free jet mode or with the glass plate as target. The gamma system of $\mathrm{NO}\left(\mathrm{NO}_{\gamma}\right)$ appears 
with weak intensity in the range from 235 to $280 \mathrm{~nm}$. NO comes from dissociation of nitrogen and oxygen molecules by electron impact, followed by recombination of the nitrogen and oxygen atoms.

$$
\mathrm{NO}\left(A^{2} \sum^{+}\right) \rightarrow \mathrm{NO}\left(X^{2} \Pi\right)+h v
$$

In addition to $\mathrm{NO}$, the copper target induces the generation of $\mathrm{N}_{2}{ }^{*}$ first positive system $(F P S)$ in the range from 600 to $800 \mathrm{~nm}$. The $\mathrm{N}_{2}{ }^{*} F S P$ emissions are due to the following processes $^{26}$ :

electron-impact excitation of the $B^{3} \Pi_{g}$ state from the $X^{l} \sum_{g}{ }^{+}$ground state:

$$
\mathrm{N}_{2}\left(X^{l} \sum_{g}^{+}\right)+\mathrm{e}^{-} \rightarrow \mathrm{N}_{2}\left(B^{3} \Pi_{g}\right)+\mathrm{e}^{-}
$$

followed by a radiative decay:

$$
\mathrm{N}_{2}\left(B^{3} \Pi_{g}\right) \rightarrow \mathrm{N}_{2}\left(A^{3} \sum_{u}^{+}\right)+h v
$$

The increase of RONS production close to the copper target surface, make the plasma jet an interesting biocide device for conductive mediums. This study demonstrates that the diagnostics of plasma jets should be applied in real conditions of the application intended when quantifying RONS. In the next part, the spatial evolutions of emission of reactive species have been made in free jet mode, and with a copper or glass plate used as the target, to determine whether modifications observed occur at the target surface or all along the plasma jet effluent.

\section{Spatial Evolution of Emission of Reactive Species}

The spatial evolution of emission intensity of various species in free jet and when copper and glass target were placed $15 \mathrm{~mm}$ from the source nozzle are presented in Fig. 7. In this study, we focused on the spatial evolution of five wavelengths: He (706 nm), O (777 $\mathrm{nm}), \mathrm{OH}(307 \mathrm{~nm}), \mathrm{N}_{2}(337 \mathrm{~nm})$, and $\mathrm{N}_{2}^{+}(391 \mathrm{~nm})$.

In free jet mode, a decrease of emission intensities for He $(706 \mathrm{~nm}), \mathrm{O}(777 \mathrm{~nm})$, and $\mathrm{OH}(307 \mathrm{~nm})$ have been observed from the source nozzle to the end of the plasma jet. The emission intensity of $\mathrm{N}_{2}(337 \mathrm{~nm})$ increases from the jet outlet and reaches its maximum intensity at $10 \mathrm{~mm}$. A similar trend was observed for the emission intensity line of $\mathrm{N}_{2}^{+}(391 \mathrm{~nm})$, with a maximum positioned at $4 \mathrm{~mm}$. For additional positions, the emissions intensities decreased. This spatial evolution of emission of reactive species for a free jet is in good agreement with previous studies. ${ }^{30}$

No significant changes in intensities range have been observed when the glass plate was used as the target (Fig. 7[b]) compared with the free mode (Fig. 7[a]). However, the presence of the glass plate modifies their evolution trend. In fact, all the emission intensities except the $\mathrm{N}_{2}(337 \mathrm{~nm})$ emission decrease from the source nozzle to 12 $\mathrm{mm}$, whereas the $\mathrm{N}_{2}(337 \mathrm{~nm})$ emission increases from the source nozzle and reaches a maximum intensity at $10 \mathrm{~mm}$, then decrease until $12 \mathrm{~mm}$. After $12 \mathrm{~mm}$, all the species increase until the target surface.

Volume 7, Issue 4, 2017 
Contrary to the results with the glass target, the use of the copper plates as the target increases the emission intensities of all species from the source nozzle until the surface target (Fig. 7[c]). This increase is more significant in the region closer to the nozzle than to the copper surface for $\mathrm{He}, \mathrm{O}, \mathrm{N}_{2}{ }^{+}$, and $\mathrm{OH}$ emissions. The $\mathrm{N}_{2}$ emission intensity increases from the source nozzle, but this increase is more intense at $10 \mathrm{~mm}$, and it continues from there to the target surface. These results are in agreement with the spectroscopy study by Yamada et al. concerning a plasma jet interaction with a copper target. ${ }^{31}$ The modifications on the spatial distribution of reactive species generated by the glass and copper targets correlate well with the local electric field formed in the region close to the target surface.

The local electric field accelerates the electrons, which increases the generation of reactive species produced directly or indirectly by electron impact reactions. This study shows, on one hand, that a conductive target generates a more intense local electric field than a nonconductive target, and on the other hand, that the increase of reactive species is due to the presence of this local electric field.

\section{CONCLUSON}

In this study, the mechanisms of plasma jet generation have been studied without a target

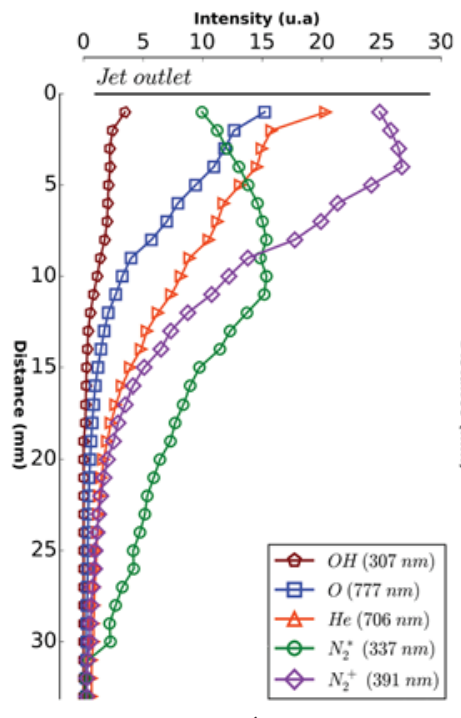

a)

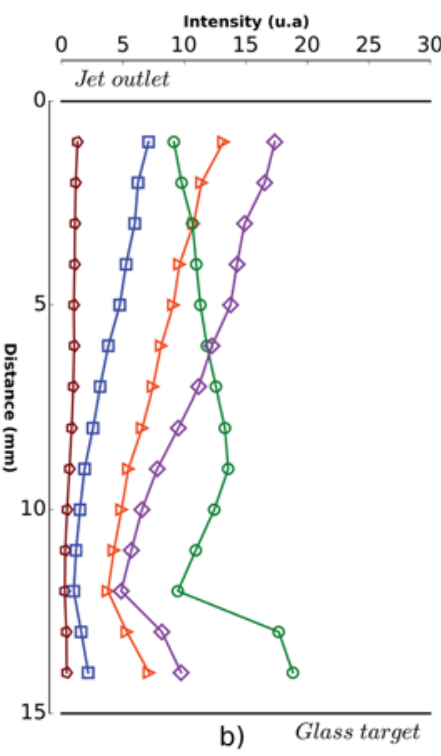

b) Glass target

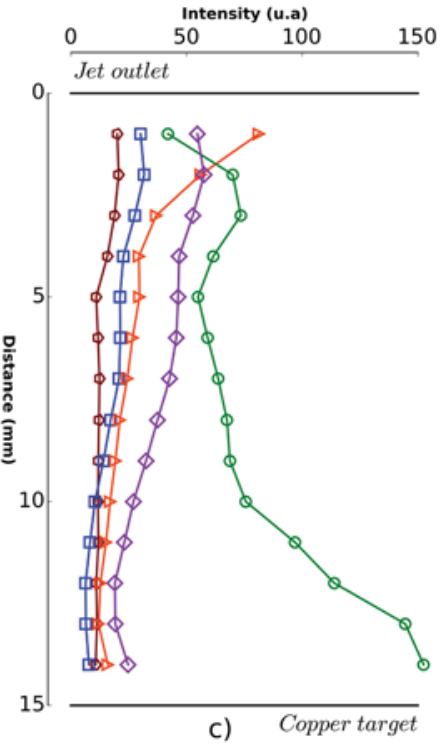

FIG. 7: Spatial evolution of plasma jet: (a) free jet, (b) with glass target, (c) with copper target. Both glass and copper targets were $15 \mathrm{~mm}$ from the source nozzle. Each spectrum was recorded with 2-sec integration time and averaging of three measurements. 
(free jet). Then, the modifications of these mechanisms by the introduction of targets in front of the plasma jet have been investigated according to the target's nature. A spreading effect of the plasma head when it interacts with a glass plate used as the dielectric target was observed. When a copper plate was used as the conductive target, a conductive channel was observed after the plasma volume strikes the target surface.

Spectroscopic studies were also carried out in order to identify the reactive species generated by the plasma jet in free jet mode. Then the modification of spectra obtained by the copper and glass targets showed the importance of the diagnostic study in real application conditions. Finally, it has been shown that the presence of local electric fields close to the target surface is responsible for modifying the spatial evolution of emission intensities of reactive species of the plasma jet.

\section{ACKNOWHDGMENT}

The authors gratefully acknowledge the Occitanie region for financial support.

\section{REFERENCES}

1. Winter J, Brandenburg R, Weltmann K-D. Atmospheric pressure plasma jets: an overview of devices and new directions. Plasma Sources Sci Technol. 2015 Dec 1;24(6):064001.

2. Laroussi M. Low-temperature plasma jet for biomedical applications: a review. IEEE Trans Plasma Sci. 2015 Mar;43(3):703-12.

3. Kong MG, Kroesen G, Morfill G, Nosenko T, Shimizu T, van Dijk J, Zimmermann JL. Plasma medicine: an introductory review. New J Phys. 2009;11(11):115012.

4. Wu S, Wang Z, Huang Q, Tan X, Lu X, Ostrikov K. Atmospheric-pressure plasma jets: Effect of gas flow, active species, and snake-like bullet propagation. Phys Plasmas. 2013 Feb 1;20(2):023503.

5. Zheng Y, Wang L, Ning W, Jia S. Schlieren imaging investigation of the hydrodynamics of atmospheric helium plasma jets. J Appl Phys. 2016 Mar 28;119(12):123301.

6. Uchida G, Takenaka K, Setsuhara Y. Influence of voltage pulse width on the discharge characteristics in an atmospheric dielectric-barrier-discharge plasma jet. Jpn J Appl Phys. 2016 Jan 1;55(1S):01 AH03.

7. Boeuf J-P, Yang LL, Pitchford LC. Dynamics of a guided streamer ('plasma bullet') in a helium jet in air at atmospheric pressure. J Phys D: Appl Phys. 2013;46(1):015201.

8. Liu X, Liu D, Cheng H, Lu X. Numerical study on propagation mechanism and bio-medicine applications of plasma jet. High Volt. 2016 Jul 1;1(2):62-73.

9. Xiong Z, Kushner MJ. Atmospheric pressure ionization waves propagating through a flexible high aspect ratio capillary channel and impinging upon a target. Plasma Sources Sci Technol. 2012 Jun 1;21(3):034001.

10. Wang L, Zheng Y, Jia S. Numerical study of the interaction of a helium atmospheric pressure plasma jet with a dielectric material. Phys Plasmas. 2016 Oct;23(10):103504.

11. Abuzairi T, Okada M, Bhattacharjee S, Nagatsu M. Surface conductivity dependent dynamic behaviour of an ultrafine atmospheric pressure plasma jet for microscale surface processing. Appl Surf Sci. 2016 Dec;390:489-96.

12. Bauville G, Pasquiers S, Blin-Simiand N, Bournonville B, Es-sebbar E, Fleury M, Jeanney P, SantosSousa J. An atmospheric argon plasma jet and its interaction with a dielectric surface. 22nd International Symposium on Plasma Chemistry. 2015 Jul [cited 2016 Nov 14]. Available from: http://www. ispc-conference.org/ispcproc/ispc22/P-II-4-9.pdf.

13. Hofmann S, van Gils K, van der Linden S, Iseni S, Bruggeman P. Time and spatial resolved optical and

Volume 7, Issue 4, 2017 
electrical characteristics of continuous and time modulated RF plasmas in contact with conductive and dielectric substrates. Eur Phys J D [serial on the Internet]. 2014 Mar [cited 2016 Nov 15];68(3). Available from: http://link.springer.com/10.1140/epjd/e2014-40430-3.

14. Liu C-T, Wu C-J, Lin Z-H, Wu J-Y, Wu J-S. Production enhancement of reactive oxygen and nitrogen species at interface of helium plasma jet and agar. IEEE Trans Plasma Sci. 2016;1-5.

15. Chauvet L, Thérèse L, Caillier B, Guillot P. Characterization of an asymmetric DBD plasma jet source at atmospheric pressure. J Anal At Spectrom. 2014 Oct 9;29(11):2050-7.

16. Wang T, Yang B, Chen X, Wang X, Yang C, Liu J. Distinct modes in the evolution of interaction between polymer film and atmospheric pressure plasma jet. Plasma Process Polym [serial on the Internet]. 2016 Sep [cited 2016 Nov 15]. Available from: http://doi.wiley.com/10.1002/ppap.201600067.

17. Lu X, Laroussi M. Dynamics of an atmospheric pressure plasma plume generated by submicrosecond voltage pulses. J Appl Phys. 2006 Sep 15;100(6):063302.

18. Jiang C, Chen MT, Gundersen MA. Polarity-induced asymmetric effects of nanosecond pulsed plasma jets. J Phys D: Appl Phys. 2009;42(23):232002.

19. Shao T, Yang W, Zhang C, Fang Z, Zhou Y, Schamiloglu E. Temporal evolution of atmosphere pressure plasma jets driven by microsecond pulses with positive and negative polarities. EPL. 2014;107(6):65004.

20. Dawson GA, Winn WP. A model for streamer propagation. Z Phys. 1965;183(2):159-171.

21. Naidis GV. Simulation of streamers propagating along helium jets in ambient air: Polarity-induced effects. Appl Phys Lett. 2011 Apr 4;98(14):141501.

22. Naidis GV. Modelling of plasma bullet propagation along a helium jet in ambient air. J Phys D: Appl Phys. 2011;44(21):215203.

23. Norberg SA, Johnsen E, Kushner MJ. Helium atmospheric pressure plasma jets touching dielectric and metal surfaces. J Appl Phys. 2015;118(1):013301.

24. Yan W, Economou DJ. Simulation of a non-equilibrium helium plasma bullet emerging into oxygen at high pressure (250-760 Torr) and interacting with a substrate. J Appl Phys. 2016 Sep 28;120(12):123304.

25. Guaitella $\mathrm{O}$, Sobota A. The impingement of a $\mathrm{kHz}$ helium atmospheric pressure plasma jet on a dielectric surface. J Phys D: Appl Phys. 2015;48(25):255202.

26. Cicala G, Tommaso ED, Rainò AC, Lebedev YA, Shakhatov VA. Study of positive column of glow discharge in nitrogen by optical emission spectroscopy and numerical simulation. Plasma Sources Sci Technol. 2009; 18(2):025032.

27. Pouvesle JM, Bouchoule A, Stevefelt J. Modeling of the charge transfer afterglow excited by intense electrical discharges in high pressure helium nitrogen mixtures. J Chem Phys. 1982 Jul 15;77(2):81725.

28. Phelps A. Tabulations of collision cross sections and calculated transport and reaction coefficients for electron collisions with $\mathrm{O}_{2}$. Boulder (CO): JILA Information Center, University of Colorado;1985 Sept. Report No: 28.

29. Popov NA. Fast gas heating in a nitrogen-oxygen discharge plasma: I. Kinetic mechanism. J Phys D: Appl Phys. 2011;44(28):285201.

30. Begum A, Laroussi M, Pervez MR. Atmospheric pressure He-air plasma jet: breakdown process and propagation phenomenon. AIP Adv. 2013 Jun 1;3(6):062117.

31. Yamada H, Sakakita H, Kato S, Kim J, Kiyama S, Fujiwara M, Itagaki H, Okazaki T,Ikehara S, Nakanishi H, Shimizu N, Ikehara Y. Spectroscopy of reactive species produced by low-energy atmosphericpressure plasma on conductive target material surface. J Phys D: Appl Phys. 2016;49(39):394001. 